\title{
Qualitative Properties of Solutions of a Doubly Nonlinear Reaction-Diffusion System with a Source
}

\author{
Mersaid Aripov $^{1}$, Shakhlo A. Sadullaeva ${ }^{2}$ \\ ${ }^{1}$ Department of Informatics and Applied Programming, National University of Uzbekistan, Tashkent, Uzbekistan \\ ${ }^{2}$ Department of Multimedia Technology, Tashkent University of Information Technology, Tashkent, Uzbekistan \\ Email: mirsaidaripov@mail.ru, sadullaeva sh@list.ru, orif sh@list.ru
}

Received 14 March 2015; accepted 5 September 2015; published 8 September 2015

Copyright (C) 2015 by authors and Scientific Research Publishing Inc.

This work is licensed under the Creative Commons Attribution International License (CC BY). http://creativecommons.org/licenses/by/4.0/

cC) (i) Open Access

\section{Abstract}

In this paper, we study properties of solutions to doubly nonlinear reaction-diffusion systems with variable density and source. We demonstrate the possibilities of the self-similar approach to studying the qualitative properties of solutions of such reaction-diffusion systems. We also study the finite speed of propagation (FSP) properties of solutions, an asymptotic behavior of the compactly supported solutions and free boundary asymptotic solutions in quick diffusive and critical cases.

\section{Keywords}

\section{Double Nonlinear Reaction-Diffusion Equation, Self-Similar Solution, Asymptotics}

\section{Introduction}

Let's consider properties of the Cauchy problem for the following system of nonlinear reaction-diffusion equations in the domain $Q=\left\{(t, x): t>0, x \in R^{N}\right\}$

$$
\begin{gathered}
\frac{\partial u}{\partial t}=\operatorname{div}\left(|x|^{k} u^{m_{1}-1}|\nabla u|^{p-2} \nabla u\right)+\gamma(t) v^{\beta_{1}} \\
\frac{\partial v}{\partial t}=\operatorname{div}\left(|x|^{k} v^{m_{2}-1}|\nabla v|^{p-2} \nabla v\right)+\gamma(t) u^{\beta_{2}}, \\
u(0, x)=u_{0}(x) \geq 0 \\
v(0, x)=v_{0}(x) \geq 0, x \in R^{N},
\end{gathered}
$$


where $k \in R, m_{1}, m_{2}>1, \beta_{1}, \beta_{2} \geq 1, p \geq 2$ are given positive numbers, $\nabla(\cdot)=\operatorname{grad}(\cdot)$ and $u_{0}(x) \geq 0, v_{0}(x) \geq 0$, $0<\gamma(t) \in C(0,+\infty)$. System (1) describes different physical process in two cormponential inhomogeneous nonlinear environments. For example, the processes of the reaction-diffusion, heat conductivity, polytrophic filtration of liquids and gas with a source power which is equal to $v^{\beta_{1}}, u^{\beta_{2}}$. Cases, when $k=l, p=2, m_{1}=m_{2}=0$, were considered in [1]-[7].

The system (1) in the domain, where $u=v=0$ is degenerated, and in the domain of degeneration it may not have the classical solution. Therefore, we study the weak solutions of system (1) which also have physical sense: $0 \leq u, v \in C(Q)$ and $|x|^{k} u^{m_{1}-1}|\nabla u|^{p-2} \nabla u,|x|^{k} v^{m_{2}-1}|\nabla v|^{p-2} \nabla v \in C(Q)$ satisfy some integral identity in the sense of distribution [1]. For the solution of system (1) there are phenomena of the finite speed of a propagation (FSP). That is, there are functions $l_{1}(t), l_{2}(t)$ that satisfy $u(t, x) \equiv 0$ and $v(t, x) \equiv 0$ at $|x| \geq l_{1}(t)$ and $|x| \geq l_{2}(t)$. In the case of $l_{1}(t), l_{2}(t)<\infty$ for $\forall t>0$, a solution of problems (1), (2) is called space localization of a disturbance. The surfaces $|x|=l_{1}(t)$ and $|x|=l_{2}(t)$ are called a free boundary or a front, respectively.

The process of the reaction-diffusion with double nonlinearity in the case of one equation has been investigated by many authors (see [8]-[15] and the references therein). FSP and blow-up property for equations with variable density

$$
\rho(x) \frac{\partial u}{\partial t}=\operatorname{div}\left(u^{m-1}|\nabla u|^{p-2} \nabla u\right),(x, t) \in R^{N+1}, \rho(x)=|x|^{-l}, l \geq 0
$$

was established in [8] [9]. An asymptotic of self-similar solutions was studied in [15]. Martynenko and Tedeev [10] [11] studied the Cauchy problem for the following two equations with variable coefficients:

$$
\rho(x) u_{t}=\operatorname{div}\left(u^{m-1}|\nabla u|^{p-2} \nabla u\right)+u^{\beta}, x \in R^{N}, t>0,
$$

and

$$
\rho(x) u_{t}=\operatorname{div}\left(u^{m-1}|\nabla u|^{p-2} \nabla u\right)+\rho(x) u^{\beta}, x \in R^{N}, t>0,
$$

where $p>1, m+p-3>0, \beta>m+p-2, \rho(x)=|x|^{-n}$, or $\rho(x)=(1+|x|)^{-n}$.

They showed that under some restrictions to the parameters and initial data, any nontrivial solution to the Cauchy problem blows up in finite time. Moreover, the authors established a sharp universal estimate of the solution near the blow-up point.

It is well know that qualitative properties of solutions of the equation similar to (1) have not been investigated thoroughly. There are some results in [1]-[6] corresponding to the case $p=2$.

In the present work, the qualitative properties of solutions of system (1) are studied based on the self-similar and approximately self-similar approach. We establish one way of construction of the critical exponent and property finite speed of perturbation (FSP) for system (1). An asymptotic property of compactly supported solutions (c.s.s.) of the considered problem and the behavior of the free boundary for the case $m_{i}+p-3>0, i=1,2$ are obtained. We prove the existence of solution with finite property. An asymptotic of a self-similar solution for the fast diffusion case $\left(m_{i}+p-3<0, i=1,2\right)$ and a critical case $m_{i}+p-3=0, i=1,2$ are also studied.

\section{Approximate Self-Similar and Self-Similar Equations}

Below we provide a method of nonlinear splitting for construction of self-similar and approximately self-similar equation. For construction of the self-similar and approximately self-similar solutions of system (1) we search the solutions $u(t, x), v(t, x)$ in the form

$$
\left\{\begin{array}{l}
u(t, x)=\bar{u}(t) w(\tau(t), \varphi(|x|)), \\
v(t, x)=\bar{v}(t) z(\tau(t), \varphi(|x|)) .
\end{array}\right.
$$

Here, we obtain $\bar{u}(t), \bar{v}(t)$ as

$$
\begin{aligned}
& \bar{u}(t)=A\left[T+\int_{0}^{t} \gamma(y) \mathrm{d} y\right]^{-\left(\beta_{1}+1\right) /\left(\beta_{1} \beta_{2}-1\right)}, \\
& \bar{v}(t)=B\left[T+\int_{0}^{t} \gamma(y) \mathrm{d} y\right]^{-\left(\beta_{2}+1\right) /\left(\beta_{1} \beta_{2}-1\right)},
\end{aligned}
$$


Which are the solutions of following equations

$$
\frac{\mathrm{d} \bar{u}}{\mathrm{~d} t}=\gamma(t) \bar{v}^{\beta_{1}}, \frac{\mathrm{d} \bar{v}}{\mathrm{~d} t}=\gamma(t) \bar{u}^{\beta_{2}} .
$$

Substituting (3), the system (1) is reduced to the following system of equations

$$
\left\{\begin{array}{l}
\frac{\partial w}{\partial \tau}=\varphi^{1-s} \frac{\partial}{\partial \varphi}\left(\varphi^{s-1} w^{m_{1}-1}\left|\frac{\partial w}{\partial \varphi}\right|^{p-2} \frac{\partial w}{\partial \varphi}\right)+\gamma(t) \bar{v}^{-\left(m_{2}+p-3\right)} \bar{u}^{\beta_{1}}\left(w+z^{\beta_{1}}\right), \\
\frac{\partial z}{\partial \tau}=\varphi^{1-s} \frac{\partial}{\partial \varphi}\left(\varphi^{s-1} z^{m_{2}-1}\left|\frac{\partial z}{\partial \varphi}\right|^{p-2} \frac{\partial z}{\partial \varphi}\right)+\gamma(t) \bar{u}^{-\left(m_{1}+p-3\right)} \bar{v}^{\beta_{2}}\left(z+w^{\beta_{2}}\right),
\end{array}\right.
$$

where the functions $\tau(t), \varphi(x)$ are chosen as following

$$
\begin{aligned}
\tau(t)= & \int_{0}^{t} \bar{u}^{m_{1}+p-3}(\eta) \mathrm{d} \eta=\int_{0}^{t} \bar{v}^{m_{2}+p-3}(\eta) \mathrm{d} \eta, \quad \text { if } m_{i}+p-3 \neq 0, i=1,2, \\
\tau(t)= & T+t, \text { if } m_{i}+p-3=0, i=1,2, \\
& \varphi(r)=\frac{1}{p_{1}}|r|^{p_{1}},|r|=\sqrt{\sum_{i=1}^{N} x_{i}^{2}}, p_{1}=\frac{p-k}{p}, s=p \frac{N}{p-k}, k<p .
\end{aligned}
$$

It is easy to establish that the system (4) has approximately self-similar solution of kind

$$
w(\tau, \varphi)=f(\xi), z(\tau, \varphi)=\psi(\xi)
$$

where $\xi=\frac{\varphi(r)}{\tau^{1 / p}}$ and the functions $f(\xi), \psi(\xi)$ satisfies the approximately self-similar system equations

$$
\begin{aligned}
& \xi^{1-s} \frac{\mathrm{d}}{\mathrm{d} \xi}\left(\xi^{s-1} f^{m_{1}-1}\left|\frac{\mathrm{d} f}{\mathrm{~d} \xi}\right|^{p-2} \frac{\mathrm{d} f}{\mathrm{~d} \xi}\right)+\frac{\xi}{p} \frac{\mathrm{d} f}{\mathrm{~d} \xi}+\gamma(t) \tau \bar{u}^{-\left(m_{1}+p-2\right)} \bar{v}^{\beta_{1}}\left(-f+\psi^{\beta_{1}}\right)=0 \\
& \xi^{1-s} \frac{\mathrm{d}}{\mathrm{d} \xi}\left(\xi^{s-1} \psi^{m_{2}-1}\left|\frac{\mathrm{d} \psi}{\mathrm{d} \xi}\right|^{p-2} \frac{\mathrm{d} \psi}{\mathrm{d} \xi}\right)+\frac{\xi}{p} \frac{\mathrm{d} \psi}{\mathrm{d} \xi}+\gamma(t) \tau \bar{v}^{-\left(m_{2}+p-2\right)} \bar{u}^{\beta_{2}}\left(-\psi+f^{\beta_{2}}\right)=0 .
\end{aligned}
$$

It is easy to prove that as $t \rightarrow \infty$

$$
\begin{aligned}
& \gamma(t) \tau \bar{u}^{-\left(m_{1}+p-2\right)} \bar{v}^{\beta_{1}} \rightarrow c_{1}, \\
& \gamma(t) \tau \bar{v}^{-\left(m_{2}+p-2\right)} \bar{u}^{\beta_{2}} \rightarrow c_{2},
\end{aligned}
$$

for $0<\gamma(t) \in H$, where $H$-Hardy's body [2], $c_{1}, c_{2}$ are constants. In this case, it is easy to show that system (1) becomes a self-similar for a sufficient large $t$. Therefore it is possible to consider the system (7) as an asymptotically self-similar system of equation corresponding to system (1). In particular case, when $\gamma(t)=$ const approximately self-similar systems (7) will be as self-similar if

$$
\left(\beta_{1}+1\right)\left(m_{1}+p-3\right)=\left(\beta_{2}+1\right)\left(m_{2}+p-3\right) .
$$
form

In this case for the functions $f(\xi), \psi(\xi)$ we have the following self-similar system of equation in "radial"

$$
\begin{aligned}
& \xi^{1-s} \frac{\mathrm{d}}{\mathrm{d} \xi}\left(\xi^{s-1} f^{m_{1}-1}\left|\frac{\mathrm{d} f}{\mathrm{~d} \xi}\right|^{p-2} \frac{\mathrm{d} f}{\mathrm{~d} \xi}\right)+\frac{\xi}{p} \frac{\mathrm{d} f}{\mathrm{~d} \xi}+a_{1}\left[-f+\psi^{\beta_{1}}\right]=0 \\
& \xi^{1-s} \frac{\mathrm{d}}{\mathrm{d} \xi}\left(\xi^{s-1} \psi^{m_{2}-1}\left|\frac{\mathrm{d} \psi}{\mathrm{d} \xi}\right|^{p-2} \frac{\mathrm{d} \psi}{\mathrm{d} \xi}\right)+\frac{\xi}{p} \frac{\mathrm{d} \psi}{\mathrm{d} \xi}+a_{2}\left[-\psi+f^{\beta_{2}}\right]=0
\end{aligned}
$$


where

$$
\begin{aligned}
& a_{1}=\left[\left(\beta_{1} \beta_{2}-1\right)-\left(m_{1}+p-3\right)\left(\beta_{1}+1\right)\right] /\left(\beta_{1} \beta_{2}-1\right), \\
& a_{2}=\left[\left(\beta_{1} \beta_{2}-1\right)-\left(m_{2}+p-3\right)\left(\beta_{2}+1\right)\right] /\left(\beta_{1} \beta_{2}-1\right) .
\end{aligned}
$$

In the case $p=2$ or $m=1$ in (10), the properties of the different solutions as computing aspects of the system Equation (10) were studied by many authors [8]-[15]. In singular, one equation case, when $\beta=m+p-2$ the existence of positive solutions of the Equation (10) was studied in [14].

\section{Slowly Diffusion Case: $m_{i}+p-3>0, i=1,2$}

\subsection{A Global Solvability of Solutions}

We prove properties of a global solvability of weak solutions of the system (1) using a comparison principle (see [1]). For this goal we construct a new system of equation using the standard equation method as in [3]:

$$
\begin{aligned}
& u_{+}(t, x)=(T+t)^{\alpha_{1}} \bar{f}(\xi), \\
& v_{+}(t, x)=(T+t)^{\alpha_{2}} \bar{\psi}(\xi),
\end{aligned}
$$

where $\quad \alpha_{i}=-\frac{\beta_{i}+1}{\beta_{i} \beta_{3-i}-1}, \quad \xi=\frac{\varphi(|x|)}{[\tau(t)]^{1 / p}}, \quad \tau(t)=\frac{1}{\lambda_{1}}(T+t)^{\lambda_{1}}, \quad \lambda_{i}=1-\alpha_{i}\left(m_{i}+p-3\right), \quad i=1,2$.

In the case, $\alpha_{1}\left(m_{1}+p-3\right)=\alpha_{2}\left(m_{2}+p-3\right)$

$$
\bar{f}(\xi)=\left(a-\xi^{p /(p-1)}\right)_{+}^{q_{2}}, \bar{\psi}(\xi)=\left(a-\xi^{p /(p-1)}\right)_{+}^{q_{1}},
$$

where $q_{1}=\frac{p-1}{m_{1}+p-3}, q_{2}=\frac{p-1}{m_{2}+p-3}, a>0,(b)_{+}=\max (0, b)$.

Fujita type critical exponent for the system (1) is numerical parameters for which the following equality holds:

$$
\left(\beta_{i}+1\right) /\left(\beta_{i} \beta_{3-i}-1\right)=N /\left[p+\left(p+m_{i}-3\right) N\right], \quad i=1,2 .
$$

This result consists of the result of Escobedo, Herero [15] for the case when $k=0, p=2, p+m_{i}-3=0, i=1,2$ in (1).

Theorem 1. (A global solvability). Assume $k<p, m_{i}+p-3>0$,

$$
\begin{gathered}
\beta_{i}>\frac{p+m_{3-i}-3}{p+m_{i}-3}, i=1,2, \\
-\frac{N}{p}+\frac{a_{1}\left(\beta_{1}+1\right)}{\beta_{1} \beta_{2}-1}+a_{1} a^{q_{2} \beta_{1}-q_{1}} \leq 0, \\
-\frac{N}{p}+\frac{a_{2}\left(\beta_{2}+1\right)}{\beta_{1} \beta_{2}-1}+a_{2} a^{q_{1} \beta_{2}-q_{2}} \leq 0, \\
u_{+}(0, x) \geq u_{0}(x), v_{+}(0, x) \geq v_{0}(x), x \in R^{N} .
\end{gathered}
$$

Then for sufficiently small $u_{0}(x), v_{0}(x)$ the followings holds

$$
u(t, x) \leq A_{1} u_{+}(t, x), v(t, x) \leq A_{2} v_{+}(t, x) \text { in } Q,
$$

where the functions $u_{+}(t, x), v_{+}(t, x)$ defined as above, $A_{1}>0, A_{2}>0$ are constants.

Proof. For proving theorem 1 we use a comparison principle. As a comparison solution we take the functions $A_{1} u_{+}(t, x), A_{2} v_{+}(t, x)$, where $A_{1}>0, A_{2}>0$.

It is easy to check that 


$$
\begin{aligned}
& \bar{u}^{-\left(m_{1}+p-2\right)} A\left(A_{1} u_{+}, A_{2} v_{+}\right)=\left[-A_{1}^{m_{1}+p-3}\left(\gamma \gamma_{1}\right)^{p-1} N+a_{1} \frac{\beta_{1}+1}{\beta_{1} \beta_{2}-1}\right] \bar{f}+a_{1} A_{2}^{\beta_{1}} \bar{\psi}^{\beta_{1}}, \\
& \bar{v}^{-\left(m_{2}+p-2\right)} B\left(A_{1} u_{+}, A_{2} v_{+}\right)=\left[-A_{2}^{m_{2}+p-3}\left(\gamma \gamma_{2}\right)^{p-1} N+a_{2} \frac{\beta_{2}+1}{\beta_{1} \beta_{2}-1}\right] \bar{\psi}+a_{2} A_{1}^{\beta_{2}} \bar{f}^{\beta_{2}},
\end{aligned}
$$

If $A_{1}^{m_{1}+p-3}\left(\gamma q_{1}\right)^{p-1}=1 / p, A_{2}^{m_{2}+p-3}\left(\gamma q_{2}\right)^{p-1}=1 / p$.

Then we have

$$
\begin{aligned}
& \bar{u}^{-\left(m_{1}+p-2\right)} A\left(A_{1} u_{+}, A_{2} v_{+}\right)=\left[-\frac{N}{p}+a_{1} \frac{\beta_{1}+1}{\beta_{1} \beta_{2}-1}\right] \bar{f}+a_{1} A_{2}^{\beta_{1}} \bar{\psi}^{\beta_{1}}, \\
& \bar{v}^{-\left(m_{2}+p-2\right)} B\left(A_{1} u_{+}, A_{2} v_{+}\right)=\left[-\frac{N}{p}+a_{2} \frac{\beta_{2}+1}{\beta_{1} \beta_{2}-1}\right] \bar{\psi}+a_{2} A_{1}^{\beta_{2}} \bar{f}^{\beta_{2}} .
\end{aligned}
$$

In order to apply a comparison principle we note that $A\left(u_{+}, v_{+}\right) \leq 0, \quad B\left(u_{+}, v_{+}\right) \leq 0$ in $D=\left\{(t, x): t>0,|x| \leq l(t)=a^{(p-1) / p}[\tau(t)]^{1 / p}\right\}$. Since

$$
\begin{aligned}
& \bar{\psi}^{\beta_{1}}(\xi) \bar{f}(\xi)=\left(a-\xi^{p /(p-1)}\right)^{\beta_{1} q_{2}-q_{1}}=\exp \left(-\left[(p-1) \frac{\beta_{1}}{m_{2}+p-3}-\frac{1}{m_{1}+p-3}\right] \eta\right) \\
& \bar{f}^{\beta_{2}}(\xi) \bar{\psi}(\xi)=\left(a-\xi^{p /(p-1)}\right)^{\beta_{2} q_{1}-q_{2}}=\exp \left(-\left[(p-1) \frac{\beta_{2}}{m_{1}+p-3}-\frac{1}{m_{2}+p-3}\right] \eta\right) .
\end{aligned}
$$

Therefore,

$$
\max \left(\bar{\psi}^{\beta_{1}}(\xi) \bar{f}(\xi)\right)=a^{\beta_{1} q_{2}-q_{1}}, \max \left(\bar{f}^{\beta_{2}}(\xi) \bar{\psi}(\xi)\right)=a^{\beta_{2} q_{1}-q_{2}} .
$$

Then according to the hypotheses of Theorem 1 and comparison principle we have

$$
u(t, x) \leq A_{1} u_{+}(t, x), v(t, x) \leq A_{2} v_{+}(t, x) \text { in } Q,
$$

if

$$
A_{1} u_{+}(0, x) \geq u_{0}(x), A_{2} v_{+}(0, x) \geq v_{0}(x), x \in R^{N} .
$$

The proof of the theorem is complete.

We notice that if

$$
\frac{\beta_{i}+1}{\beta_{i} \beta_{3-i}-1}=\frac{N}{p+\left(p+m_{i}-3\right) N}, i=1,2
$$

then

$$
\begin{aligned}
& \bar{u}^{-\left(m_{1}+p-2\right)} A\left(A_{1} u_{+}, A_{2} v_{+}\right)=A_{2}^{\beta_{1}} \bar{\psi}^{\beta_{1}} \geq 0, \\
& \bar{v}^{-\left(m_{2}+p-2\right)} B\left(A_{1} u_{+}, A_{2} v_{+}\right)=A_{1}^{\beta_{2}} \bar{f}^{\beta_{2}} \geq 0 .
\end{aligned}
$$

It means that

$$
u(t, x) \geq A_{1} u_{+}(t, x), v(t, x) \geq A_{2} v_{+}(t, x) \text { in } Q,
$$

if $u_{0}(x) \geq A_{1} u_{+}(0, x), v_{0}(x) \geq A_{2} v_{+}(0, x), x \in R^{N}$.

\subsection{Property of Finite Speed of a Perturbation}

Corollary 1. Suppose that the hypotheses of Theorem 1 holds. Then a solution of the problems (1), (2) has FSP property.

Indeed, for a weak solution of the problems (1), (2) we have

$$
u(t, x) \leq A_{1} u_{+}(t, x), v(t, x) \leq A_{2} v_{+}(t, x) \text { in } Q,
$$


It follows that

$$
u(t, x) \equiv 0, v(t, x) \equiv 0, \text { in } Q / D,
$$

where $D=\left\{(x, t): t>0,|x|<a^{(p-1) / p}[\tau(t)]^{1 /(p-k)}\right\}$. It means that the solution of the problems (1), (2) have FSP property.

Critical case. The case $m_{i}+p-3<0, i=1,2$ will be called a critical case.

Theorem 2. Let $k<p, m_{i}+p-3<0, i=1,2, \frac{\beta_{1}+1}{\beta_{1} \beta_{2}-1}<\frac{N}{p}, \frac{\beta_{2}+1}{\beta_{1} \beta_{2}-1}<\frac{N}{p}$. Then for sufficiently small $u_{0}(x), v_{0}(x)$ the problems (1), (2) have global solution and the following inequalities in $Q$ hold

$$
\begin{aligned}
& u(t, x) \leq(T+t)^{-N / p} \exp \left(-\left(\frac{\xi^{p}}{p}\right)\right), \\
& v(t, x) \leq(T+t)^{-N / p} \exp \left(-\left(\frac{\xi^{p}}{p}\right)\right),
\end{aligned}
$$

here $\xi=\frac{\varphi(|x|)}{[\tau(t)]^{1 / p}}$,

Proof. Proof of the theorem is based on the comparison principle. We take for comparison the functions

$$
\begin{aligned}
& u_{+}(t, x)=B_{1}(T+t)^{-N / p} \bar{f}(\xi), \\
& v_{+}(t, x)=B_{2}(T+t)^{-N / p} \bar{\psi}(\xi),
\end{aligned}
$$

where $\bar{f}(\xi)=\exp \left(-\left(\frac{\xi^{p}}{p}\right)\right), \bar{\psi}(\xi)=\exp \left(-\left(\frac{\xi^{p}}{p}\right)\right), \xi=\frac{|\eta|}{(T+t)^{1 / p}}, B_{i}>0, i=1,2$.

It is easy to check that

$$
\begin{aligned}
& \bar{u}^{-\left(m_{1}+p-2\right)} A\left(B_{1} u_{+}, B_{2} v_{+}\right)=\left[-\frac{N}{p}+\frac{\beta_{1}+1}{\beta_{1} \beta_{2}-1}\right]+B_{2}^{\beta_{1}} \bar{\psi}^{\beta_{2}-1} \psi^{\beta_{1}}, \\
& \bar{v}^{-\left(m_{2}+p-2\right)} B\left(B_{1} u_{+}, B_{2} v_{+}\right)=\left[-\frac{N}{p}+\frac{\beta_{1}+1}{\beta_{1} \beta_{2}-1}\right]+B_{1}^{\beta_{2}} \bar{f}^{\beta_{1}-1} f^{\beta_{2}} .
\end{aligned}
$$

From the hypothesis of Theorem 2 and last expressions we have

$$
\begin{aligned}
& A\left(B_{1} u_{+}, B_{2} v_{+}\right) \leq 0, \\
& B\left(B_{1} u_{+}, B_{2} v_{+}\right) \leq 0 \text { in } Q,
\end{aligned}
$$

if the constants $B_{1}, B_{2}$ such that

$$
B_{1}^{\beta_{2}} \leq-\frac{N}{p}+\frac{\beta_{1}+1}{\beta_{1} \beta_{2}-1}, \quad B_{2}^{\beta_{1}} \leq-\frac{N}{p}+\frac{\beta_{2}+1}{\beta_{1} \beta_{2}-1} .
$$

This inequality due to the comparison principle completes the proof of the theorem.

Value $\beta_{1}, \beta_{2}$ for which

$$
\begin{aligned}
& \frac{N}{p}=\frac{\beta_{1}+1}{\beta_{1} \beta_{2}-1}, \\
& \frac{N}{p}=\frac{\beta_{2}+1}{\beta_{1} \beta_{2}-1},
\end{aligned}
$$

corresponds to Fujita type critical exponent proved earlier by Escobedo, Herrero [15] for the case $p=2$. 


\section{Asymptotic of the Self-Similar Solutions}

Now we study asymptotic of the weak compact supported solutions (c.s.s.) of the system (10) when $\gamma(t)=$ const. Consider this system equation with boundary condition

$$
\begin{aligned}
& f(0)=c_{1}>0, f(d)=0, \\
& \psi(0)=c_{2}>0, \psi(d)=0,
\end{aligned}
$$

where $0<d<+\infty$.

The existence of a self-similar weak c.s. solution for the problems (10), (15) in the case $k=0, p=2$ was studied in [6] where the authors obtained conditions for existence of the c.s. solution.

We seek solution of the system (10) in the form

$$
\begin{aligned}
& f(\xi)=\bar{f}(\xi) y_{1}(\eta), \eta=-\ln \left(a-\xi^{\frac{p}{p-1}}\right), \\
& \psi(\xi)=\bar{\psi}(\xi) y_{2}(\eta), \eta=-\ln \left(a-\xi^{\frac{p}{p-1}}\right),
\end{aligned}
$$

where

$$
\begin{aligned}
& \bar{f}(\xi)=\left(a-\xi^{\gamma}\right)_{+}^{q_{1}}, \bar{\psi}(\xi)=\left(a-\xi^{\gamma}\right)_{+}^{q_{2}}, a>0, \\
& q_{1}=\frac{p-1}{p+m_{1}-3}, q_{2}=\frac{p-1}{p+m_{2}-3}, \gamma=\frac{p}{p-1} .
\end{aligned}
$$

Theorem 3. Assume that $q_{1}>0, q_{2}>0, \beta_{1} q_{2}>q_{1}, \beta_{2} q_{1}>q_{2}$. Then the weak compactly support solutions (c.s.s) $f(\xi), \psi(\xi)$ of the system (10) as $\eta \rightarrow \infty\left(\eta=-\ln \left(a-\xi^{p /(p-1)}\right)\right)$ has asymptotic

$$
\begin{aligned}
& f(\xi)=c_{1} \bar{f}(\xi), \\
& \psi(\xi)=c_{2} \bar{\psi}(\xi),
\end{aligned}
$$

where the coefficients $c_{1}, c_{2}$ satisfied to system of the algebraic equations

$$
\begin{aligned}
& -q_{1}^{p-1} c_{1}^{m_{1}+p-3}+a_{1} \gamma^{-p}=0, \\
& -q_{2}^{p-1} c_{2}^{m_{2}+p-3}+a_{2} \gamma^{-p}=0 .
\end{aligned}
$$

Proof. It is easy to check that

$$
\begin{gathered}
\xi^{N-1} \bar{f}^{m_{1}-1}\left|\frac{\mathrm{d} \bar{f}}{\mathrm{~d} \xi}\right|^{p-2} \frac{\mathrm{d} \bar{f}}{\mathrm{~d} \xi}+\xi^{N}\left(\gamma q_{1}\right)^{p-1} \quad \bar{f} \in C(0, \infty), \\
\xi^{N-1} \bar{\psi}^{m_{2}-1}\left|\frac{\mathrm{d} \bar{\psi}}{\mathrm{d} \xi}\right|^{p-2} \frac{\mathrm{d} \bar{\psi}}{\mathrm{d} \xi}+\left(\gamma q_{2}\right)^{p-1} \xi^{N} \quad \bar{\psi} \in C(0, \infty)
\end{gathered}
$$

and

$$
\begin{aligned}
& \xi^{1-N} \frac{\mathrm{d}}{\mathrm{d} \xi}\left(\xi^{N-1} \bar{f}^{m_{1}-1}\left|\frac{\mathrm{d} \bar{f}}{\mathrm{~d} \xi}\right|^{p-2} \frac{\mathrm{d} \bar{f}}{\mathrm{~d} \xi}\right)=-\left(\gamma q_{1}\right)^{p-1}\left(N \bar{f}+\xi \frac{\mathrm{d} \bar{f}}{\mathrm{~d} \xi}\right) \\
& \xi^{1-N} \frac{\mathrm{d}}{\mathrm{d} \xi}\left(\xi^{N-1} \bar{\psi}^{m_{2}-1}\left|\frac{\mathrm{d} \bar{\psi}}{\mathrm{d} \xi}\right|^{p-2} \frac{\mathrm{d} \bar{\psi}}{\mathrm{d} \xi}\right)=-\left(\gamma q_{2}\right)^{p-1}\left(N \bar{\psi}+\xi \frac{\mathrm{d} \bar{\psi}}{\mathrm{d} \xi}\right), \quad \gamma=\frac{p}{p-1} .
\end{aligned}
$$

We will show that the functions $\bar{f}(\xi), \bar{\psi}(\xi)$ should be main member of asymptotic of solution of the system (10). For this goal we search the solution of system (10) in the form 


$$
\begin{aligned}
f(\xi) & =\bar{f}(\xi) w(\eta), \\
\psi(\xi) & =\bar{\psi}(\xi) z(\eta), \quad \eta=-\ln \left(a-\xi^{p /(p-1)}\right) .
\end{aligned}
$$

By using expression (10) it is easy to cheek that

$$
\begin{gathered}
\xi^{s-1} f^{m_{1}-1}\left|\frac{\mathrm{d} f}{\mathrm{~d} \xi}\right|^{p-2} \frac{\mathrm{d} f}{\mathrm{~d} \xi}=\gamma^{p-1} \xi^{s} \bar{f} L_{1}(w), \\
L_{1}(w)=w^{m_{1}-1}\left|\frac{\mathrm{d} w}{\mathrm{~d} \eta}-q_{1} w\right|^{p-2}\left(\frac{\mathrm{d} w}{\mathrm{~d} \eta}-q_{1} w\right), \\
\xi^{s-1} \psi^{m_{2}-1}\left|\frac{\mathrm{d} \psi}{\mathrm{d} \xi}\right|^{p-2} \frac{\mathrm{d} \psi}{\mathrm{d} \xi}=\gamma^{p-1} \xi^{s} \bar{\psi} L_{2}(z), \\
L_{2}(z)=z^{m_{2}-1}\left|\frac{\mathrm{d} z}{\mathrm{~d} \eta}-q_{2} z\right|^{p-2}\left(\frac{\mathrm{d} z}{\mathrm{~d} \eta}-q_{2} z\right), \\
\xi^{1-s} \frac{\mathrm{d}}{\mathrm{d} \xi}\left(\xi^{s-1} f^{m_{1}-1}\left|\frac{\mathrm{d} f}{\mathrm{~d} \xi}\right|^{p-2} \frac{\mathrm{d} f}{\mathrm{~d} \xi}\right)=\gamma^{p-1} \bar{f}\left[\left(s-q_{1} \gamma \frac{\xi^{\gamma}}{a-\xi^{\gamma}}\right) L_{1} w+\gamma \frac{\xi^{\gamma}}{a-\xi^{\gamma}} \frac{\mathrm{d}}{\mathrm{d} \eta} L_{1} w\right] \\
\xi^{1-s} \frac{\mathrm{d}}{\mathrm{d} \xi}\left(\xi^{s-1} \psi^{m_{2}-1}\left|\frac{\mathrm{d} \psi}{\mathrm{d} \xi}\right|^{p-2} \frac{\mathrm{d} \psi}{\mathrm{d} \xi}\right)=\gamma^{p-1} \bar{\psi}\left[\left(s-q_{2} \gamma \frac{\xi^{\gamma}}{a-\xi^{\gamma}}\right) L_{2} z+\gamma \frac{\xi^{\gamma}}{a-\xi^{\gamma}} \bar{\psi} \frac{\mathrm{d}}{\mathrm{d} \eta} L_{2} z\right] .
\end{gathered}
$$

Therefore according transformation (16) the system (10) reduced to the system

$$
\begin{aligned}
& \frac{\mathrm{d}}{\mathrm{d} \eta} L_{1}(w)+\left(\frac{s}{\gamma} \varphi_{1}(\eta)-q_{1}\right) L_{1}(w)+\frac{1}{p} \gamma^{-p} \varphi_{1}(\eta)\left(\frac{\mathrm{d} w}{\mathrm{~d} \eta}-q_{1} w\right) \\
& -\frac{\beta_{1}+1}{\beta_{1} \beta_{2}-1} \gamma^{-p} \varphi_{1}(\eta) w+\gamma^{-p} \frac{\mathrm{e}^{\left(-q_{1}+\beta_{1} q_{2}-1\right) \eta}}{a-\mathrm{e}^{-\eta}} z^{\beta_{1}}=0, \\
& \frac{\mathrm{d}}{\mathrm{d} \eta} L_{2}(z)+\left(\frac{s}{\gamma} \varphi_{1}(\eta)-q_{2}\right) L_{2}(z)+\frac{1}{p} \gamma^{-p} \varphi_{1}(\eta)\left(\frac{\mathrm{d} z}{\mathrm{~d} \eta}-q_{2} z\right) \\
& -\frac{\beta_{2}+1}{\beta_{1} \beta_{2}-1} \gamma^{-p} \varphi_{1}(\eta) z+\gamma^{-p} \frac{\mathrm{e}^{\left(-q_{2}+\beta_{2} q_{1}-1\right) \eta}}{a-\mathrm{e}^{-\eta}} w^{\beta_{2}}=0,
\end{aligned}
$$

where $\varphi_{1}(\eta)=\frac{\mathrm{e}^{-\eta}}{a-\mathrm{e}^{-\eta}}$.

Analysis of solution of last system shows that $w \rightarrow c_{1}, z \rightarrow c_{2}$, as $\eta \rightarrow \infty$, where constants $c_{1}, c_{2}$ are the solutions of the algebraic system equations

$$
\begin{aligned}
& -q_{1}^{p-1} c_{1}^{m_{1}+p-3}+a_{1} \gamma^{-p}=0, \\
& -q_{2}^{p-1} c_{2}^{m_{2}+p-3}+a_{2} \gamma^{-p}=0 .
\end{aligned}
$$

The proof of the theorem is complete.

5. Quick Diffusion Case: $m_{i}+p-3<0, i=1,2$

Theorem 4. Let $m_{i}+p-3<0, i=1,2$. Then regular (quenching) solution of the system (10) as $\xi \rightarrow \infty$ has asymptotic

$$
\begin{aligned}
& f(\xi)=c_{3}\left(a+\xi^{p /(p-1)}\right)^{q_{1}}, \\
& \psi(\xi)=c_{4}\left(a+\xi^{p /(p-1)}\right)^{q_{2}} .
\end{aligned}
$$


Here

1) if $-q_{1}+\beta_{1} q_{2}=0,-q_{2}+\beta_{2} q_{1}=0$, then the coefficients $c_{3}, c_{4}$ are the roots of the nonlinear system of the algebraic equations

$$
\begin{aligned}
& \gamma^{p-1}\left(\frac{p N}{(p-k)+\gamma q_{1}}\right) c_{3}^{m_{1}+p-2}+\left(a_{1} q_{1}+\frac{\beta_{1}+1}{\beta_{1} \beta_{2}-1}\right) c_{3}+c_{4}^{\beta_{1}}=0, \\
& \gamma^{p-1}\left(\frac{p N}{(p-k)+\gamma q_{2}}\right) c_{4}^{m_{2}+p-2}+\left(a_{2} q_{2}+\frac{\beta_{2}+1}{\beta_{1} \beta_{2}-1}\right) c_{4}+c_{3}^{\beta_{2}}=0 .
\end{aligned}
$$

2) if $\beta_{1} q_{2}+1<q_{1}, \beta_{2} q_{1}+1<q_{2}$, then the coefficients $c_{3}, c_{4}$ are the roots of the nonlinear system of the algebraic equations

$$
\begin{aligned}
& \gamma^{p-1}\left(\frac{p N}{(p-k)+\gamma q_{1}}\right) c_{3}^{m_{1}+p-3}+\left(a_{1} q_{1}+\frac{\beta_{1}+1}{\beta_{1} \beta_{2}-1}\right)=0, \\
& \gamma^{p-1}\left(\frac{p N}{(p-k)+\gamma q_{2}}\right) c_{4}^{m_{2}+p-3}+\left(a_{2} q_{2}+\frac{\beta_{2}+1}{\beta_{1} \beta_{2}-1}\right)=0 .
\end{aligned}
$$

Proof. We will seek a solution of system (10) in following form

$$
\begin{aligned}
& f(\xi)=\tilde{f}(\xi) w(\eta), \\
& \psi(\xi)=\tilde{\psi}(\xi) z(\eta), \eta=\ln \left(a+\xi^{p /(p-1)}\right) .
\end{aligned}
$$

Since

$$
\begin{aligned}
& \xi^{1-s} \frac{\mathrm{d}}{\mathrm{d} \xi}\left(\xi^{s-1} f^{m_{1}-1}\left|\frac{\mathrm{d} f}{\mathrm{~d} \xi}\right|^{p-2} \frac{\mathrm{d} f}{\mathrm{~d} \xi}\right)=\gamma^{p-1} \bar{f}\left(s+q_{1} \gamma \frac{\xi^{\gamma}}{a+\xi^{\gamma}}\right) L_{1} w+\gamma \frac{\xi^{\gamma}}{a+\xi^{\gamma}} \bar{f} \frac{\mathrm{d}}{\mathrm{d} \eta} L_{1} w \\
& \xi^{1-s} \frac{\mathrm{d}}{\mathrm{d} \xi}\left(\xi^{s-1} \psi^{m_{2}-1}\left|\frac{\mathrm{d} \psi}{\mathrm{d} \xi}\right|^{p-2} \frac{\mathrm{d} \psi}{\mathrm{d} \xi}\right)=\gamma^{p-1} \bar{\psi}\left(s+q_{2} \gamma \frac{\xi^{\gamma}}{a+\xi^{\gamma}}\right) L_{2} z+\gamma \frac{\xi^{\gamma}}{a+\xi^{\gamma}} \bar{\psi} \frac{\mathrm{d}}{\mathrm{d} \eta} L_{2} z .
\end{aligned}
$$

By substituting (21) into (10) we get

$$
\begin{aligned}
& \frac{\mathrm{d}}{\mathrm{d} \eta} L_{1}(w)+\left(\frac{s}{\gamma} \varphi_{2}(\eta)+q_{1}\right) L_{1}(w)+a_{1} \gamma^{-p}\left(\frac{\mathrm{d} w}{\mathrm{~d} \eta}+q_{1} w\right) \\
& +\frac{\beta_{1}+1}{\beta_{1} \beta_{2}-1} \gamma^{-p} \varphi_{2}(\eta) w+\gamma^{-p} \varphi_{2}(\eta) \mathrm{e}^{\left(-q_{1}+\beta_{1} q_{2}\right) \eta} \mathrm{z}^{\beta_{1}}=0 \\
& \frac{\mathrm{d}}{\mathrm{d} \eta} L_{2}(z)+\left(\frac{s}{\gamma} \varphi_{2}(\eta)+q_{2}\right) L_{2}(z)+a_{2} \gamma^{-p}\left(\frac{\mathrm{d} z}{\mathrm{~d} \eta}+q_{2} z\right) \\
& +\frac{\beta_{2}+1}{\beta_{1} \beta_{2}-1} \gamma^{-p} \varphi_{2}(\eta) z+\gamma^{-p} \varphi_{2}(\eta) \mathrm{e}^{\left(-q_{2}+\beta_{2} q_{1}\right) \eta} w^{\beta_{2}}=0
\end{aligned}
$$

where $\varphi_{2}(\eta)=\frac{\mathrm{e}^{\eta}}{a+\mathrm{e}^{\eta}}$.

Analyzing of solutions system (22) when $\eta \rightarrow \infty$ we conclude that the solutions of this system $w \rightarrow c_{3}, z \rightarrow c_{4}$, where constants $c_{3}, c_{4}$ are solutions of the algebraic system (19), (20).

\section{References}

[1] Samarskii, A.A., Galaktionov, V.A., Kurdyumov, S.P. and Mikhailov, A.P. (1995) Blow-Up in Quasilinear Parabolic Equations. Berlin, 4, Walter de Grueter, 535. http://dx.doi.org/10.1515/9783110889864

[2] Aripov, M. (2000) Asymptotic of the Solution of the Non-Newton Polytropical Filtration Equation. Journal of Applied Mathematics and Mechanics, 80, 767-768. 
[3] Aripov, M. (1988) Method of the Standard Equation for the Solution of the Nonlinear Value Problem. Fan, Tashkent, $137 \mathrm{p}$.

[4] Aripov, M. and Sadullaeva, Sh.A. (2013) To Properties of the Equation of Reaction Diffusion with Double Nonlinearity and Distributed Parameters. Journal of Siberian Federal University. Mathematics \& Physics, 6, 157-167.

[5] Aripov, M. and Sadullaeva, Sh.A. (2010) To Solutions of One No Divergent Type Parabolic Equation with Double Nonlinearity. Progress in Analysis and Its Applications, 12-18.

[6] Aripov, M. and Muhammadiev, J. (1999) Asymptotic Behavior of an Automodel Solutions for One System of Quasilinear Equations of Parabolic Type Romania. Buletini Stiintific, Seria Matematicasi Informatica, No. 3, 19-40.

[7] Aripov, M., Khaydarov, A. and Sadullaeva, Sh.A. (2007) Numerical Modeling of Some Processes in a Nonlinear Moving Media. J. Source of Knowledge, No. 1, 20-26.

[8] Martynenko, A.V. and Tedeev, A.F. (2007) The Cauchy Problem for a Quasilinear Parabolic Equation with a Source and Inhomogeneous Density. Computational Mathematics and Mathematical Physics, 47, 238-248. http://dx.doi.org/10.1134/S096554250702008X

[9] Martynenko, A.V. and Tedeev, A.F. (2008) On the Behavior of Solutions to the Cauchy Problem for a Degenerate Parabolic Equation with Inhomogeneous Density and a Source. Computational Mathematics and Mathematical Physics, 48, 1145-1160. http://dx.doi.org/10.1134/S0965542508070087

[10] Tedeyev, A.F. (2004) Conditions for the Existence and Nonexistence of a Compact Support in Time of Solutions of the Cauchy Problem for Quasilinear Degenerate Parabolic Equations. Siberian Mathematical Journal, 45, 189-200. http://dx.doi.org/10.1023/b:simj.0000013021.66528.b6

[11] Martinenko, A.V. and Shramenko, V.N. (2010) Estimate of solutions of the Cauchy Problem near the Time of Exacerbation for a Quasilinear Parabolic Equation with a Source and a Variable Density. Nonlinear Boundary Value Problems, 20, 104-115.

[12] Zheng, P., Mu, Ch., Liu, D., Yao, X. and Zhou, Sh. (2012) Blow-Up Analysis for a Quasilinear Degenerate Parabolic Equation with Strongly Nonlinear Source. Abstract and Applied Analysis, 2012, Article ID: 109546, 19 p.

[13] Dimova, S.N., Kastchiev, M.S., Koleva, M.G. and Vasileva, D.P. (1995) Numerical Analysis of the Blow-Up Regimes of Combustion of Two-Component Nonlinear Heat-Conducting Medium. Journal of Computational Mathematics and Mathematical Physics, 35, 380-399.

[14] Kurduomov, S.P., Kurkina, E.S. and Telkovskaya, O.V. (1989) Blow-Up in Two Componentional Environment. Mathematical Modeling, 1, 34-50.

[15] Escobedo, M. and Herrero, M.A. (1991) Boundedness and Blow up for a Semi Linear Diffusion Reaction System. Journal of Differential Equations, 89, 176-192. http://dx.doi.org/10.1016/0022-0396(91)90118-S 